\title{
Population Pharmacokinetics, Efficacy Exposure-response Analysis, and Model-based Meta-analysis of Fenebrutinib in Subjects with Rheumatoid Arthritis
}

\author{
Phyllis Chan ' • Jiajie Yu' - Leslie Chinn ' Marita Prohn ${ }^{2} \cdot$ Jan Huisman ${ }^{2} \cdot$ Brett Matzuka $^{3}$. \\ William Hanley ${ }^{4} \cdot$ Katie Tuckwell $^{5} \cdot$ Angelica Quartino' $^{\prime}$
}

Received: 19 October 2019 / Accepted: 18 December 2019 / Published online: 6 January 2020

(C) The Author(s) 2020, corrected publication 2020

\begin{abstract}
Purpose Fenebrutinib (GDC-0853), a Bruton's tyrosine kinase (BTK) inhibitor was investigated in a Phase 2 clinical trial in patients with rheumatoid arthritis (RA). Our aim was to apply a model-informed drug development (MIDD) approach to examine the totality of available clinical efficacy data.

Methods Population pharmacokinetics (popPK) modeling, exposure-response (E-R) analysis, and model-based metaanalysis (MBMA) of fenebrutinib were performed based on the Phase 2 data.

Results PopPK of fenebrutinib after oral administration was described using a 3-compartment model with linear elimination and a flexible absorption transit compartment model. Healthy subjects had a 52\% higher apparent clearance than patients. E-R analyses based on longitudinal ACR20, ACR50, and ACR70 and DAS28 (CRP) data modeled fenebrutinib effect with an $\mathrm{E}_{\max }$ function, and an efficacy plateau was
\end{abstract}

The original version of this article was revised: The article was published with an incomplete title. The complete title is "Population Pharmacokinetics, Efficacy Exposure-response Analysis, and Model-based Meta-analysis of Fenebrutinib in Subjects with Rheumatoid Arthritis".

Electronic supplementary material The online version of this article (https://doi.org/l 0. I007/s I | 095-0 19-2752-y) contains supplementary material, which is available to authorized users.

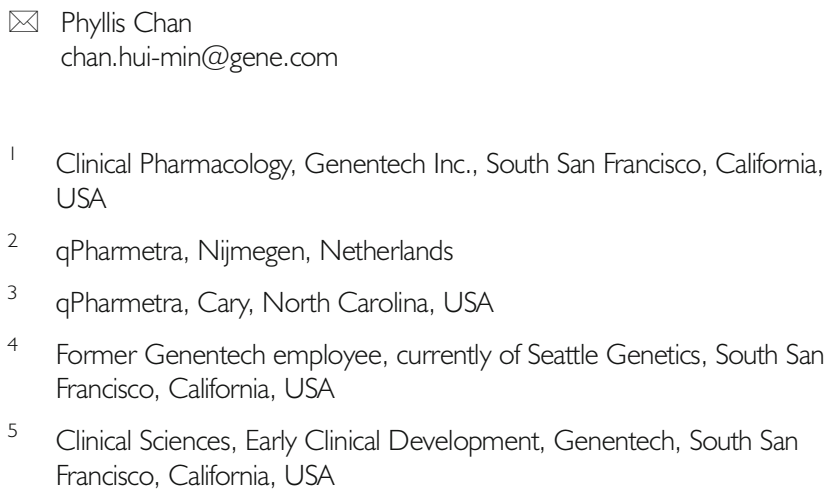

achieved within the exposure range obtained in the Phase 2 clinical trial. Based on literature data, a summary-level clinical efficacy database was constructed, and MBMA determined ACR20, ACR50, and ACR70 responder rates in the placebo and adalimumab arms of the Phase 2 clinical trial were found to be consistent with historical data for these treatments.

Conclusions Our multi-pronged approach applied MIDD to maximize knowledge extraction of efficacy data and enabled robust interpretation from a Phase 2 clinical trial.

KEY WORDS Bruton's tyrosine kinase (BTK) inhibitor . exposure-response $\cdot$ model-based meta-analysis $\cdot$ population pharmacokinetics $\cdot$ rheumatoid arthritis

$\begin{array}{ll}\text { ABBREVIATIONS } \\ \text { AUC } & \text { Area under the concentration-time curve } \\ \text { BTK } & \text { Bruton's tyrosine kinase } \\ \text { CL/F } & \text { Apparent clearance } \\ \text { C }_{\max } & \text { Maximum concentration } \\ \text { C }_{\min } & \text { Trough concentration } \\ \text { CRP } & \text { C-reactive protein } \\ \text { DAS28 } & \text { Disease Activity Score with 28-joint counts } \\ \text { EBE } & \text { Empirical Bayes estimates } \\ \text { E-R } & \text { Exposure-response } \\ \text { FI } & \text { Relative extent of absorption } \\ \text { FOCE-I } & \text { First order conditional estimation with interaction } \\ \text { MBMA } & \text { Model-based meta-analysis } \\ \text { MIDD } & \text { Model-informed drug development } \\ \text { MTT } & \text { Mean transit time } \\ \text { MTX } & \text { Methotrexate } \\ \text { NLME } & \text { Non-linear mixed effects } \\ \text { NTR } & \text { Number of transit compartments } \\ \text { PCVPC } & \text { Prediction-corrected visual predictive check } \\ \text { PD } & \text { Pharmacodynamic }\end{array}$


PK Pharmacokinetics

popPK Population pharmacokinetics

PPC Posterior predictive check

PPI Proton pump inhibitor

RA Rheumatoid arthritis

RF Rheumatoid factor

SLE Systemic lupus erythematosus

TCAM Transit compartment absorption model

$T_{\max } \quad$ Time associated with maximum concentration

VPC Visual predictive check

\section{INTRODUCTION}

\section{Model Informed Drug Development (MIDD)}

In 2017, the sixth iteration of the Prescription Drug User Fee Act (PDUFA VI) was authorized as part of the FDA Reauthorization Act (FDARA). Among its goals was to augment the Agency's expertise in model-informed drug development (MIDD) approaches and support the evaluation of model-based strategies used to guide development efforts (1).

MIDD approaches can be extremely informative to drug development: they can be used to characterize the exposureresponse (E-R) relationship of new drugs, especially when the relationship is complex and its interpretation challenging, and to support selection of the appropriate dose and/or regimen for an upcoming clinical trial. These approaches result in more powerful inferences than other, non-integrated analyses as they help to fully identify various aspects of the E-R relationship and maximize knowledge when harvesting from the drug's proof of concept study by examining the totality of clinical efficacy and safety data. Drug development in rheumatoid arthritis (RA) patients can be used to illustrate the use of MIDD approaches (2) in particular, where a large number of studies showing the efficacy of therapy across various mechanisms of action have been published, thus providing the ability to pool data and make salient comparisons.

\section{Bruton's Tyrosine Kinase (BTK) and Fenebrutinib}

RA is an autoimmune disorder, and although several therapeutics are approved for the treatment of patients with an inadequate response to methotrexate (MTX) or tumor necrosis factor inhibitors (anti-TNF) (3-9), some patients become refractory to currently available therapeutics and may benefit from therapy utilizing a different mechanism of action (6,8-12).

Bruton's tyrosine kinase (BTK) is a nonreceptor Tec family tyrosine kinase broadly expressed in hematopoietic cells (except $\mathrm{T}$ cells) and plays a crucial role in signaling through the $\mathrm{B}$ cell antigen receptor and the $\mathrm{Fc} \gamma$ receptor $(\mathrm{Fc} \gamma \mathrm{R})$ in $\mathrm{B}$ cells and myeloid cells, respectively (13-16). Therefore, the inhibition of BTK represents an attractive potential therapeutic approach for the treatment of immunological disorders such as RA or systemic lupus erythematosus (SLE) (15-20), in which B cells and myeloid cells induce or sustain an excessive autoimmune response. However, currently there is no approved BTKtargeted therapy for such chronic autoimmune indications.

Fenebrutinib (GDC-0853, RG7845) is an orally administered BTK inhibitor that is highly selective and noncovalent, leading to reversible binding, intended to block B cell proliferation and the resulting excessive immune response seen in autoimmune disorders (21). Fenebrutinib has previously been evaluated in healthy subjects (22) and patients with resistant B cell lymphoma or chronic lymphocytic leukemia (23).

The pharmacokinetics (PK) of fenebrutinib was characterized previously in healthy subjects in a Phase 1 trial, and plasma concentrations peaked $1-3 \mathrm{~h}$ after oral administration and declined thereafter, with a steady-state half-life ranging from 4.2-9.9 h (22). In the Phase 1 trial, fenebrutinib plasma exposures were found to increase approximately doseproportionally with modest accumulation following twice daily dosing. Additionally, dose- and concentration-dependent inhibition of BTK was observed, and pharmacokinetic/ pharmacodynamic (PK/PD) models were developed using the PD biomarkers data to describe the treatment effect of fenebrutinib on BTK inhibition, as assessed by BTK autophosphorylation on circulating B cells and basophils in healthy volunteers. Subsequent simulations conducted with these PK/ PD models suggest that a once-daily dosing regimen would maintain steady-state plasma concentrations associated with a high degree of CD63 inhibition over the entire dosing interval.

Fenebrutinib is currently being investigated in patients with RA, chronic spontaneous urticaria, and SLE. To fully understand the treatment effect of fenebrutinib in patients with RA, an MIDD approach was implemented by investigating the totality of clinical efficacy data from a Phase 2 trial through the integration of population pharmacokinetics (popPK) modeling, E-R analysis, and model-based meta-analysis (MBMA) of fenebrutinib.

\section{MATERIALS AND METHODS}

\section{Trial Design}

GA29350 (ANDES, ClinicalTrials.gov Identifier: NCT02833350) was a multicenter, Phase 2, randomized, double-blind, placebo-controlled, parallel-group, doseranging trial to evaluate the efficacy and safety of fenebrutinib in patients with moderate to severe active RA and an inadequate response to previous MTX therapy (cohort 1) or antiTNF therapy (cohort 2) (24,25). All patients were seropositive for either rheumatoid factor (RF) or anti-citrullinated peptide 
antibody, or had a history of seropositivity upon entry into the trial and were on stable doses of MTX during the trial.

In cohort 1, 480 patients were enrolled, and were randomly assigned to 1 of 5 parallel treatment arms ( 3 fenebrutinib arms, 1 placebo arm, and 1 adalimumab arm). In the fenebrutinib and placebo arms, subjects received $50 \mathrm{mg}$ QD, $150 \mathrm{mg}$ QD, or $200 \mathrm{mg}$ BID fenebrutinib tablets or placebo tablets, together with subcutaneous $(\mathrm{SC})$ placebo injections every other week for 12 weeks $(n=40$ in the $50 \mathrm{mg}$ arm, and $n=109$ to 111 in each of the other 4 arms). In the active comparator arm, patients received adalimumab $40 \mathrm{mg} \mathrm{SC}$ every other week and placebo tablets for 12 weeks. In cohort $2(n=98$ enrolled), patients received $200 \mathrm{mg}$ fenebrutinib BID or placebo tablets for 12 weeks.

Fenebrutinib dose selection for the Phase 2 trial was based on its safety profile, PK properties, and the expectation that plasma concentrations achieved would reach 70 to $90 \%$ BTK inhibition over the dosing interval in the majority of patients. Three dose levels were selected for cohort 1 to obtain a broad range of fenebrutinib exposures, with minimal exposure overlap among the dose groups, to allow a thorough investigation of E-R in patients. As the lowest fenebrutinib dose (50 mg QD) in the trial was not expected to achieve maximal efficacious response, a reduced number of subjects were assigned to this dose group.

Fenebrutinib plasma concentrations were determined by high performance liquid chromatography with tandem mass spectrometry (MS/MS) detection (Covance Laboratories Inc., USA). Calibration plots were linear throughout the range of 0.5 to $500 \mathrm{ng} / \mathrm{mL}$ (lower and upper limits of quantification, respectively) for fenebrutinib in human plasma. Relative standard deviation of precision was $<7.8 \%$, and the accuracy of the method was between $91.3 \%$ and $102.7 \%$.

\section{Population Pharmacokinetic (popPK) Analysis}

Fenebrutinib plasma concentrations from subjects providing all available PK data after tablet administration in the clinical studies were integrated into the popPK analysis. The model was characterized initially in healthy subjects (22) from the Phase 1 multiple ascending dose (MAD) (GA29347) and a Phase 1 relative bioavailability/food effect/drug-drug interaction (GP29832, GlinicalTrials.gov Identifier: NCT02699710) studies, and later updated to incorporate the Phase 2 data from the three fenebrutinib-treated arms in patients with RA. PK sampling schedules of the 3 studies included in the popPK analysis are presented in Table S1.

The popPK model was developed by introducing features in the order of increasing complexity, beginning with very simple models (e.g. one-compartment with first-order elimination), and continuing until further improvement in model fit was not supported by the data.

Covariate analysis first investigated the effects of predefined demographic, laboratory, prognostic, and treatment covariates on all the model parameters by using univariate screening. If $>10 \%$ of a covariate was missing, the covariate was not included in the popPK analysis. Otherwise, the value of missing covariate observations was imputed as the median of the remaining values from an appropriate sub-population. For example, the missing baseline body weight value was imputed as the gender-adjusted median body weight of the remaining subjects. For categorical covariates, the most frequently occurring value was imputed for subjects with missing values in each trial. Concomitant proton pump inhibitor (PPI) and food were categorized as time-varying covariates, whereas the other categorical covariates were considered as baseline (non-time-varying) covariates. Next, covariates selected from the univariate screening ( $p$ value $<0.05$ ) were included in the evaluation of a full model followed by backwards elimination ( $p$ value $<0.01)$. Effects of continuous covariates were incorporated into the model using the power function and normalized using the median of the covariate. Effects of categorical covariates were parameterized as proportional effects in the model, in which the fractional change on a model parameter was estimated, and the typical category constituted the largest proportion in the analysis population.

The final model was determined on the basis of objective function value, physiological plausibility of model parameter estimates, numerical convergence success, parameter estimate precision (relative standard errors $<50 \%$ ), and visual predictive check (VPG) plots. Goodness-of-fit plots were produced to verify the general agreement of fitted values with observed data. Prediction-corrected visual predictive check (pcVPG) was performed by normalizing the observed and simulated dependent variable based on the typical population prediction for the median independent variable in the bin (26). The pcVPC was stratified to evaluate the covariate effects of interest, whenever applicable.

In addition to exploring the effects of covariates on the intersubject variability of $\mathrm{PK}$ of fenebrutinib, the popPK model was also used to generate empirical Bayes estimates (EBE; individual posthoc) for exposure, in terms of maximum concentration $\left(\mathrm{C}_{\max }\right)$, trough concentration $\left(\mathrm{C}_{\min }\right)$, and total daily area-under-the-concentration-time-curve (AUG) at steady state as predicted using nominal dose due to the high patient compliance in the trial (dose intensity rate $\geq 99.3 \%$ / arm/cohort), for the E-R analysis.

Model development was carried out using first order conditional estimation with interaction (FOCE-I) as implemented in NONMEM (version 7.3, ICON Development Solutions, Ellicott City, MD). Post-processing of NONMEM analysis results was carried out in $\mathrm{R}$ (version 3.2.2, $\mathrm{R}$ Development Core Team, 2008).

\section{Exposure-Response (E-R) Analysis}

Treatment responses in RA clinical trials are commonly evaluated by the American College of Rheumatology 
improvement criteria for 20,50, and 70\% threshold values (ACR20, ACR50, and ACR70, respectively), which is a binary response variable, and by the Disease Activity Score with 28-joint counts (DAS28), which is a continuous variable $(27,28)$. Both types of endpoints can be measured repeatedly during the course of the treatment period. The proportion of patients achieving ACR50 responses and analysis of longitudinal DAS28 (CRP) (DAS based on 28 joints and C-reactive protein value) were evaluated as the primary, key secondary, and secondary endpoints in the Phase 2 trial, and therefore, were of primary interest among clinical efficacy endpoints modeled for fenebrutinib. The E-R analyses aimed to establish the relationship between patient-specific fenebrutinib exposure with clinical efficacy endpoints of interest.

\section{ACR20, ACR50, and ACR70}

Modeling of ACR20, ACR50, and ACR70 responses in individual patients following treatment with fenebrutinib used observations from cohorts 1 and 2 from the Phase 2 trial. The E-R analysis dataset consisted of a total of 7005 observations (2335 each for ACR20, ACR50, and ACR70) from 467 patients. Observations were on days 7, 14, 28, 56, and 84 after the initiation of study treatment. Study dropout rate was low, with a majority of patients completing the 12 -week trial with $\geq 90 \%$ completion rate/arm/cohort. Non-responder imputation was used for patients who discontinued prior to week 12 , and for whom an ACR response could not be determined. Given the number of patients and use of imputation, there were 467 observations available on each observation day. Study treatments included placebo and fenebrutinib doses of $50 \mathrm{mg}$ QD, $150 \mathrm{mg}$ QD, and $200 \mathrm{mg}$ BID.

Fenebrutinib exposure metrics of $\mathrm{C}_{\max }, \mathrm{C}_{\text {min }}$, and $\mathrm{AUC}$ at steady state as the driver for the E-R relationship were explored. Graphics created with $\mathrm{R}$ were used to view the time course of fractions of ACR20, ACR50, and ACR70 responders, placebo effect, and treatment effect based on fenebrutinib exposure.

An E-R analysis was conducted with NONMEM, using Laplacian conditional estimation with -2 times the $\log$ of the likelihood (-2LL). Simultaneous modeling (29) of ACR20, ACR50, and ACR70 and all time points reflected the analysis decision to utilize the benefits of pooling data across highly interrelated observations. The E-R model considered the longitudinal measurements as a function of time, placebo effect, and fenebrutinib exposure:

\footnotetext{
logit of $A C R=$ Baseline + Time effect + Covariate effect + Markovian element Time effect $=\frac{\text { Tmax } \times \text { time }^{\text {Hill }}}{\text { time }^{\text {Hill }}+T_{50}{ }^{\text {Hill }}}$

Covariate effect $=$ Geographic region + Rheumatoid factor $+\frac{\text { Fenebrutinib } A U C}{\text { Fenebrutinib } A U C+E C_{50}}$
}

where Tmax is the maximum placebo effect over time, T50 is the time associated with $50 \%$ of placebo effect, and EC50 is the fenebrutinib exposure (in terms of AUG) associated with $50 \%$ of drug effect. Finally, an analysis of covariate effects on the endpoints was performed, and simulation $(n=1000)$ was conducted based on the final model and summarized using posterior predictive check (30).

\section{DAS28 (CRP)}

Modeling of the E-R relationship between fenebrutinib exposure and DAS28 (GRP) response included observed data from cohorts 1 and 2 of the Phase 2 trial. The analysis dataset consisted of 2676 observations from 467 patients measured on days $0,7,14,28,56$, and 84 after the initiation of study treatment. There were 467 observations at baseline, decreasing to 424 observations on day 84 . The data utilized last observation carried forward (LOCF) imputation but excluded withdrawal. As was done for E-R analysis in ACR20, ACR50, and ACR70, patient-specific predictions of exposure metrics in terms of steady state $\mathrm{C}_{\max }, \mathrm{C}_{\min }$, and $\mathrm{AUC}$ from the popPK model were used to enable $\mathrm{E}-\mathrm{R}$ modeling. In addition, the data were explored graphically prior to modeling, and an E-R analysis was performed using longitudinal DAS28 (CRP) data. Model development was conducted using first-order conditional estimation with interaction (FOCE-I) as implemented in NONMEM. The final model fit the observed data as a function of time, placebo effect, and fenebrutinib exposure. No covariate analysis was performed due to the supportive and exploratory nature of using a second efficacy endpoint, and simulation $(n=1000)$ was conducted based on the final model and summarized using visual predictive check.

\section{Model-Based Meta-Analysis (MBMA) Modeling of ACR20, ACR50, and ACR70}

An efficacy and safety meta-analysis database was constructed in July 2017 using publicly available data of randomized trials in RA, based on the guidelines in the Cochrane Handbook for Systematic reviews. For this, a systematic review of publicly available data from the PubMed, Cochrane Library, and Embase databases was conducted using the search term "rheumatoid arthritis". Furthermore, an existing database that contained data published in or before 2012 (30) was augmented using an additional, systematic, and quality-controlled procedure to search for relevant published studies, extract their data, and add to the existing database yielding pertinent data published through 2017.

The summary data of the database were explored systematically to determine the amount of data available for each treatment, efficacy and safety endpoint across trials, distribution of mean patient characteristics, and to graphically view the time course of longitudinal endpoints, placebo effect, active treatment effect from each of the comparator treatments, and the effects of 
patient and trial characteristics. Response rates of ACR20, ACR50, and ACR70 in terms of the proportion of patients achieving specified thresholds were the most prevalent efficacy endpoints in the database, and therefore, were chosen as the variables for model development using MBMA. Because of the relatively few adverse events observed in the fenebrutinib Phase 2 trial in RA (24), MBMA for safety events, such as serious infections, was not conducted.

The model simultaneously fit ACR20, ACR50, and ACR70 longitudinal data, and a non-linear mixed effects (NLME) estimation method was carried out using $\mathrm{R}$. This hierarchical regression model used maximum likelihood estimation, and multiple levels of heterogeneity were described as mixed effect variability terms accommodating between-trial and within-trial variability. A residual variability term was used to account for the unexplained deviation from fixed effects (31). The effects of time, placebo treatment, active drug dose, and additional covariates were considered during model development. For treatments where dose-ranging data were available, the potential doseresponse relationships were tested.

The adequacy of model fits across trials was evaluated by visually inspecting time course plots of observed distributions vs. predicted values. Model simulations were performed using fixed and random effect estimates from the final MBMA model to predict the treatment effects over time at hypothetical doses of fenebrutinib comparing to the approved drug dose levels, as well as to further evaluate difference in treatment response rates among various patient populations in RA.

\section{RESULTS}

\section{Population Pharmacokinetic (popPK)Modeling}

The popPK analysis dataset consisted of 130 males and 255 females, between 18 and 75 years of age, and baseline body weights ranging from 38 to $153 \mathrm{~kg}$. The demographics of the subjects included in the popPK analysis are summarized in Table S2. No imputation of PK values was performed, and records with missing $\mathrm{PK}$ or time values were omitted from the analysis. In total, $506(11 \%)$ out of 4565 observations were reported as having concentration below the lower limit of quantification (BLQ), and were excluded from the analysis without further assessment of their impact on parameter estimates. Predefined set of clinical and demographic covariate factors (Table S2) were investigated during popPK model development to assess their influence on the PK characteristics. The analysis dataset did not include C-reactive protein (CRP) values from healthy subjects in the Phase 1 multiple ascending dose (MAD) trial or race information from the Phase 1 relative bioavailability/food effect/drug-drug interaction trial.

The final popPK model was a 3-compartment model with linear elimination from the central compartment. Its parameter estimates are shown in Table I. A flexible transit compartment absorption model (TCAM) was used to describe the fenebrutinib absorption (32). The final model included effects of PPI concomitant administration, food intake, and formulation on mean transit time (MTT) between absorption compartments, number of transit compartments (NTR), and relative extent of absorption (F1). In addition, concomitant administration of PPI, age, and subject status (i.e. healthy subject or RA patient) were found to affect apparent clearance $(\mathrm{CL} / \mathrm{F})$. The residual variability was described using a proportional error model that was assumed to decrease with time via an exponential model for the Phase 1 studies and constant for Phase 2 trial, due to the sparse sampling in the Phase 2 clinical trial.

The final popPK model (Model S1) captured the observed data well and adequately described the overall fenebrutinib concentration versus time profiles. The performance and adequacy of the model were demonstrated using GOF plots (Fig. S1) and pcVPC plots (Fig. S2). Median individual post hoc exposures among the three fenebrutinib dose groups investigated in the Phase 2 trial were well separated (Fig. S3). The epsilon-shrinkage value was $12.5 \%$.

Statistically significant covariates included the impact of subject status, age, and concomitant administration of PPI on apparent clearance. Healthy subjects in the two Phase 1 studies had a 52\% higher apparent clearance than patients with RA in the Phase 2 trial. Additionally, apparent clearance $(\mathrm{CL} / \mathrm{F})$ decreases with age $(15.2 \%$ lower in a 75 -year-old patient compared to a 25-year-old patient), and concomitant administration of PPI (decreases by $33.7 \%$ ).

\section{Exposure-Response (E-R) Modeling}

\section{ACR20, ACR50, and ACR70}

The efficacy of fenebrutinib as defined by the probability of achieving ACR20, ACR50, and ACR70 was described by a logistic model that represented effects of treatments as a function of time, fenebrutinib exposure at steady state, and patient characteristics. The time component was modeled as a sigmoidal $\mathrm{E}_{\max }$ function to represent the increase in efficacy to a maximum over time. The fenebrutinib effect on response was modeled as an $\mathrm{E}_{\max }$ function driven by fenebrutinib exposure as measured by $\mathrm{C}_{\max }, \mathrm{C}_{\min }$, or $\mathrm{AUC}$, and the statistical significance of incorporating each exposure metric into the E-R model was similar in terms of $p$-values. Steady state AUC was chosen as the driver for efficacy, based on exploratory results, and prior E-R modeling knowledge in RA $(33,34)$. Separate $\mathrm{E}_{\max }$ functions to describe the E-R relationship for each of ACR20, ACR50, and ACR70 probability were tested, but it was seen that a single $\mathrm{E}_{\max }$ term for all three ACR thresholds adequately captured the data. Additionally, a Markovian element was added to reflect the inherent serial correlation in the data (29). It is notable that by definition, at time zero, all 
Table I Parameter Estimates of the Final popPK Model

\begin{tabular}{|c|c|c|c|c|c|}
\hline Parameter & Alias & Estimate & $\begin{array}{l}\text { Relative } \\
\text { SE (\%) }\end{array}$ & $95 \% \mathrm{Cl}$ & $\begin{array}{l}\text { Shrinkage } \\
(\%)\end{array}$ \\
\hline$\theta_{1}$ & CL/F: Apparent systemic clearance $\left(L \cdot h^{-1}\right)$ & 19.5 & $\ldots .$. & $(18.2-20.9)$ & \\
\hline$\theta_{2}$ & $\begin{array}{l}\text { VI/F: Apparent central volume of distribu- } \\
\text { tion (L) }\end{array}$ & 381 & $\ldots$ & $(333-437)$ & \\
\hline$\theta_{3}$ & $\begin{array}{l}\text { V2/F: Apparent peripheral volume of distri- } \\
\text { bution }(\mathrm{L})\end{array}$ & 284 & $\ldots .$. & $(254-3 \mid 8)$ & \\
\hline$\theta_{4}$ & $\begin{array}{l}\text { Q।/F: Apparent Intercompartmental clear- } \\
\text { ance }\left(L \cdot h^{-1}\right)\end{array}$ & 52.8 & $\ldots$ & $(44.1-63.2)$ & \\
\hline$\theta_{5}$ & $\begin{array}{l}\text { V3/F: Apparent second Peripheral volume } \\
\text { of distribution }(\mathrm{L})\end{array}$ & 273 & $\ldots$. & $(222-336)$ & \\
\hline$\theta_{6}$ & $\begin{array}{l}\text { Q2/F: Apparent second intercompartmen- } \\
\text { tal clearance }\left(\mathrm{L} \cdot \mathrm{h}^{-1}\right)\end{array}$ & 4.47 & $\ldots .$. & $(3.73-5.36)$ & \\
\hline$\theta_{7}$ & NTR: Number of transit compartments & 14.9 & $\ldots$. & $(13.0-17.1)$ & \\
\hline$\theta_{8}$ & MTT: Mean transit time $(h)$ & 0.849 & $\ldots .$. & $\begin{array}{l}(0.755- \\
0.954)\end{array}$ & \\
\hline$\theta_{9}$ & Proportional residual error (\%) & 1.94 & $\ldots .$. & $(1.29-2.92)$ & \\
\hline$\theta_{11}$ & Food effect on MTT & 1.43 & $\ldots .$. & $(1.22-1.68)$ & \\
\hline$\theta_{12}$ & PPI effect on MTT & 0.835 & $\ldots .$. & $(0.692-1.01)$ & \\
\hline$\theta_{13}$ & PPI and food effect on MTT & 2.26 & $\ldots$ & $(1.78-2.86)$ & \\
\hline$\theta_{14}$ & PPI effect on $\mathrm{FI}$ & 0.657 & $\cdots$ & $\begin{array}{c}(0.568- \\
0.759)\end{array}$ & \\
\hline$\theta_{15}$ & PPI and food effect on FI & 0.693 & $\ldots$ & $\begin{array}{l}(0.611- \\
0.785)\end{array}$ & \\
\hline$\theta_{16}$ & Food effect on NTR & 0.864 & $\ldots$. & $(0.45-1.66)$ & \\
\hline$\theta_{17}$ & Tablet effect on NTR & 0.049 & $\ldots .$. & $\begin{array}{c}(0.0287- \\
0.0838)\end{array}$ & \\
\hline$\theta_{18}$ & Residual error rate in healthy volunteers & 1.94 & $\ldots .$. & $(1.53-2.46)$ & \\
\hline$\theta_{19}$ & $\begin{array}{l}\text { Maximum residual error in healthy } \\
\text { volunteers }\end{array}$ & 6.66 & $\ldots .$. & $(4.21-10.6)$ & \\
\hline$\theta_{20}$ & PPI effect on $\mathrm{CL} / \mathrm{F}$ & 0.663 & $\ldots$. & $\begin{array}{l}(0.650- \\
0.675)\end{array}$ & \\
\hline$\theta_{21}$ & Proportional residual error in patients & 0.390 & $\ldots$. & $\begin{array}{l}(0.372- \\
0.408)\end{array}$ & \\
\hline$\theta_{22}$ & Age effect on CL/F & -0.161 & 41.4 & $\begin{array}{c}(-0.291-- \\
0.0304)\end{array}$ & \\
\hline$\theta_{23}$ & Healthy volunteer effect on $\mathrm{CL} / \mathrm{F}$ & 1.52 & $\ldots$. & $(1.27-1.82)$ & \\
\hline$\omega_{1.1}$ & $\omega^{2} \mathrm{CL/F}$ & 0.0732 & 14.9 & $\begin{array}{l}(0.0518- \\
0.0946)\end{array}$ & 27.3 \\
\hline$\omega_{2.2}$ & $\omega^{2} V I / F$ & 0.100 & 22.6 & $\begin{array}{l}(0.0558- \\
0.145)\end{array}$ & 54.4 \\
\hline$\omega_{8.8}$ & $\omega_{M T T}^{2}$ & 0.0861 & 29.7 & $\begin{array}{r}(0.0360- \\
0.136)\end{array}$ & 58.1 \\
\hline$\omega_{9.9}$ & $\omega_{\mathrm{Fl}}^{2}$ & 0.131 & 17.1 & $\begin{array}{r}(0.0872- \\
0.175)\end{array}$ & 31.9 \\
\hline$\omega_{10.1}$ & $\omega^{2}$ IOV on $\mathrm{FI}$ & 0.299 & 5.30 & $\begin{array}{l}(0.268- \\
0.330)\end{array}$ & 26.0 \\
\hline
\end{tabular}

Log-transformed parameters have been back-transformed and thus relative SE (\%) is not represented, instead $95 \% \mathrm{Cl}$ is appropriate

popPK population pharmacokinetics, SE standard error, Cl confidence interval, PPI proton pump inhibitor, FI absorption patients are non-responders, and thus observed baseline measurement is zero. However, during model development, it was found that including estimated baselines in the model improved the fit at later time points substantially, without unduly adversely affecting the model predictions at time zero, where it is understood that the actual result is zero probability. Therefore, separate baseline terms were estimated for each of ACR20,
ACR50, and ACR70 probability achievement, and an interindividual variability term was added on baseline (Model S2).

A clear E-R relationship was observed between fenebrutinib exposure in terms of steady state AUC and the probability of achieving ACR20, ACR50, and ACR70. A dropout model was not incorporated due to the high completion rate $(\geq 90 \%)$ of the Phase 2 trial. Table S3 shows the summary statistics of 
the list of predefined covariates explored for the E-R analysis. The subsequent univariate screening, followed by full model formation and then backward elimination, found that baseline rheumatoid factor (RF) level was a statistically significant covariate. Parameter estimates for the E-R model using ACR20, ACR50, and ACR70 data are shown in Table S4.

The final E-R model fit the data adequately as evident in the posterior predictive check (PPG) plots that assessed the performance of the models: (a) longitudinal probability of achieving ACR20, ACR50, and ACR70 stratified by fenebrutinib dose (Fig. 1), and (b) fenebrutinib exposure effect (Fig. 2). The 90\% prediction interval for the $50 \mathrm{mg}$ QD dose group is wider than the other 3 dose groups, due to the smaller number of subjects from the $50 \mathrm{mg}$ QD arm compared to the others in the Phase 2 trial. The correlation coefficients ( $r$ values) between ACR20 and ACR50, between ACR50 and ACR70, and between ACR20 and ACR70 are 0.415, 0.59, and 0.245 , respectively, for observed data, and $0.439,0.613$, and 0.269 , respectively, for model-based simulation.

\section{DAS28 (CRP)}

The longitudinal measurements of DAS28 (CRP) were described by a logistic model that includes the effects of treatments as a function of time, fenebrutinib exposure in terms of steady state AUC, and patient characteristics. The time component was incorporated as a sigmoidal $\mathrm{E}_{\max }$ function to represent efficacy increases to a maximum effect over time, with separate parameters for fenebrutinib and placebo for the time at which each reached $50 \%$ of maximum effect.

Exploration of an E-R relationship between fenebrutinib exposure, in terms of $\mathrm{C}_{\max }, \mathrm{C}_{\min }$, and $\mathrm{AUC}$ at steady state, and DAS28 (CRP) levels was performed. As with the E-R analysis conducted using ACR response data, AUC was chosen as the exposure metric with which to conduct subsequent E-R analysis using DAS28 (CRP). The fenebrutinib exposure effect was modeled as a simple $\mathrm{E}_{\max }$ function. Inter-individual variability was placed on baseline and the maximum effect parameters. The NONMEM control stream for the model and the parameter estimates are shown in Model S3 and Table S5, respectively.

The VPG plots (Fig. 3 for the timecourse of DAS28 (CRP) stratified by fenebrutinib dose; Fig. 4 for the effect of fenebrutinib exposure) were used to assess the performance of the models, and show that the final model fit the data adequately.

\section{Model-Based Meta-Analysis (MBMA) of ACR20, ACR50, and ACR70}

The meta-analysis database contained publicly available trial data from 62 citations and included 9 drugs of multiple mechanisms of action plus placebo. Notably, among these were 16 citations providing summary metrics from 1871 patients for adalimumab, 51 providing summary metrics for placebo from 6567 patients, and 10 containing summary metrics from 1132 patients for tofacitinib, collectively used to characterize both the longitudinal course of effect over time, and dose-response for drugs of interest. For each treatment, there were between 1 and 6 dose levels (median of 3), and between 1 and 47 observation times (median of 11). All of the above metrics also applied to the ACR50 subset of the database, except that it contained between 1 and 44 observation times (median 10.5). Observations from fenebrutinib Phase 2 trial were summarized at the study level and added to the database. Table S6 summarize the ACR20, ACR50, and ACR70 data, and sources from the database.

Analysis of summary-level treatment efficacy from fenebrutinib and published RA trial data was described by a logistic model, using the observed fraction of patients who achieved the three ACR improvement thresholds. The model incorporated the effect of placebo or active treatment as a function of time, as well as the effects of drug doses, and patient population characteristics. The time component was modeled as an exponential function reflecting the growth to a maximum effect, and the dose-response varied by treatment. Where appropriate, an $\mathrm{E}_{\max }$ function was used to describe the dose-response, while in others, a linear relationship or a single level of treatment effect was estimated. If data from different dosing schedules were available, the dose was normalized to total amount in one week in order to pool data for the same treatment across multiple trials, when necessary. Separate baselines were estimated for each of the ACR20, ACR50, and ACR70. The model included a function to ensure that the resulting predictions for ACR20, ACR50, and ACR70 were in proper order. Between-trial and between-arm variability was modeled on baseline, while between-drug variability was modeled on the rate of change in the time course.

The dose-response of fenebrutinib on ACR response endpoints in the MBMA model was informed by leveraging patient-level data described for E-R analysis. The dose at which $50 \%$ of efficacy $\left(\mathrm{ED}_{50}=525 \mathrm{mg} /\right.$ week) in the MBMA was calculated and fixed in the model using the modelpredicted exposure associated with $50 \%$ of efficacy $\left(\mathrm{EC}_{50}=\right.$ $2650 \mathrm{ng} * \mathrm{~h} / \mathrm{mL}$ ) that was estimated in the E-R analysis for ACR20, ACR50, and ACR70. Lastly, covariate analysis using forward addition found a high proportion $(>80 \%)$ of patients who had inadequate response to previous anti-TNF therapy (TNF-IR), the percentage of patients who had failed previous MTX treatment (MTX-IR), and concurrent MTX therapy had statistically significant impact on ACR response rates. The $\mathrm{R}$ code for the final MBMA model is shown in Data $\mathrm{S} 1$.

The ACR20, ACR50, and ACR70 response rates in the placebo and adalimumab arms of the fenebrutinib Phase 2 trial were found to be consistent with historical data for these treatments (Fig. 5). Additionally, the model performance was validated using observed response rates across the treatments used, their doses, and the longitudinal time courses of effect against $90 \%$ prediction intervals for each treatment, as seen in representative treatments in Fig. 6. 

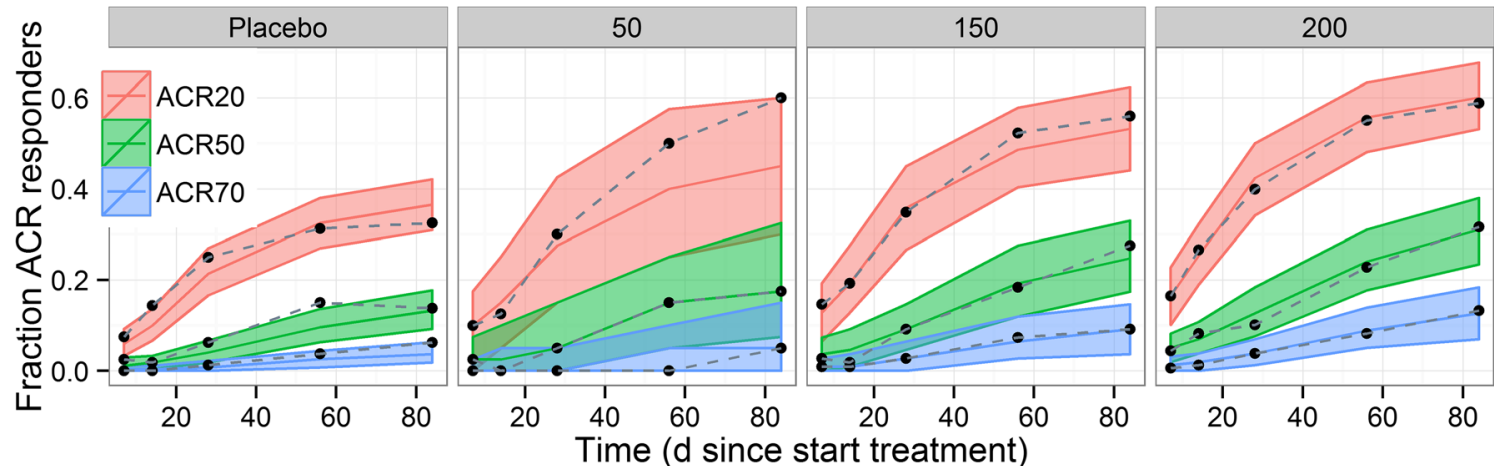

Fig. I Posterior predictive check for exposure-response model of ACR20, ACR50, and ACR70 - time course by fenebrutinib dose. Black dots: observed responder fraction; bands: 90\% prediction interval; red, green, blue middle lines: median prediction.

Leveraging data across all 10 treatments in the model, the final MBMA model estimates that populations with at least $80 \%$ of patients who are TNF-IR would have lower response than those with higher percentages of patients who had previously failed on MTX. Background use of MTX resulted in increased patient population response rates.

The utilization of comprehensive historical data through MBMA enabled comparison of fenebrutinib versus its potential comparator compounds. Simulation based on the developed MBMA model showed that $200 \mathrm{mg}$ BID fenebrutinib was predicted to have similar efficacy in terms of ACR20, ACR50, and ACR70 compared to the registrational doses of adalimumab and tofacitinib in a MTX-IR (but anti-TNFnaïve) population at 12 weeks after initiation of treatment, when incorporating variability in PK and efficacy endpoints, as depicted in Fig. 7. Simulation results for TNF-IR patient populations are included in Fig. S4 to demonstrate, as an example, the utility of applying the developed MBMA model to predict efficacy in another patient population, which showed that $200 \mathrm{mg}$ BID fenebrutinib would have comparable efficacy to the registrational doses of adalimumab and tofacitinib in terms of ACR20, ACR50, and ACR70, in TNF-IR patients at 12 weeks after initiation of treatment.

\section{DISCUSSION}

The wealth and diversity of efficacy data among various treatments in RA clinical trials necessitate an integrated approach to fully leverage knowledge from publicly available summary-level data and understand the impact of treatments based on new mechanisms of action. Therefore, we aimed to design a novel study with multiple model-based analyses using data from the fenebrutinib Phase 2 trial in patients with RA, which we report here.

\section{Population Pharmacokinetics (popPK) Analysis}

The popPK analysis conducted herein provided a useful representation of the exposures resulting from investigated doses of fenebrutinib, and the effects of patient characteristics on the inter-subject variability of the PK parameters. Food and PPI effects were incorporated into the model using TCAM, because previous knowledge using non-compartmental analysis from the Phase 1 relative bioavailability/food effect/drugdrug interaction study indicated that food blunts the concentration-time profile and delays the $\mathrm{T}_{\max }$, whereas PPI decreases the $\mathrm{C}_{\max }$. In addition, out of the other statistically significant covariates in the final popPK model, covariate exploration found that the age and subject status in the analysis dataset could be confounded; i.e. healthy subjects tend to be younger than patients with RA. Shrinkage values for interindividual variability and residual error terms in the popPK model are within an acceptable range, and along with the results from EBE-based model diagnostic methods, these indicate the observed data provided sufficient information for individual posthoc exposures estimation for the E-R analyses. Finally, if a larger sample size were available, additional
Fig. 2 Posterior predictive check for exposure-response model of ACR20, ACR50, and ACR70 - by fenebrutinib AUC bins at week 12 . AUC, area under the concentration time curve. Black dots: observed responder fraction at fenebrutinib exposure quartiles; bands: $90 \%$ prediction interval; red, green, blue middle lines: median prediction.
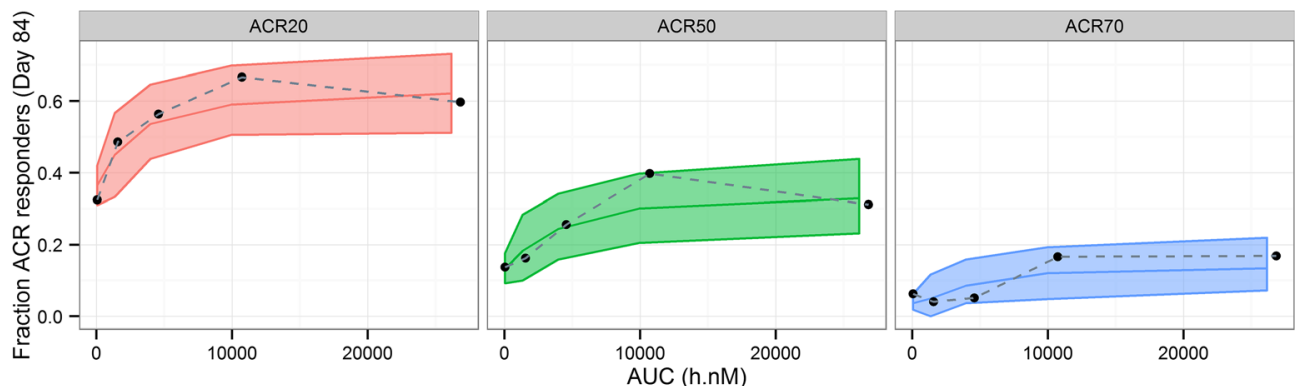


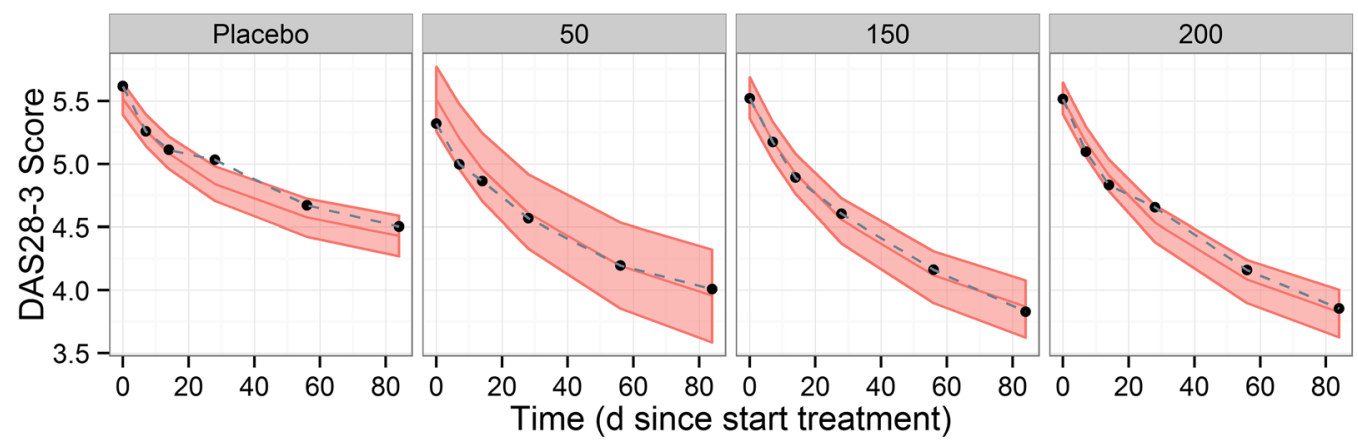

Fig. 3 Visual predictive check of DAS28 (CRP) exposure-response model - time course by fenebrutinib dose. Black dots: observed responder fraction; bands: 90\% prediction interval; red middle lines: median prediction.

causes of inter-subject variability in patients could be further investigated.

\section{Exposure-Response (E-R) Analyses}

The primary goals of the E-R analyses with fenebrutinib were to characterize the relationship between exposure and clinical efficacy endpoints of ACR responses and DAS28 (CRP) levels, and to determine whether the efficacy could be further augmented with increasing exposure or dose. The longitudinal data of both ACR responses and DAS28 (CRP) levels were modeled. Graphical exploration and model-based simulation suggest that a plateau of efficacy was achieved within the exposure range obtained in the study, and further increase in fenebrutinib dose would not lead to additional efficacy in cohort 1 patients who were MTX-IR. The lack of difference in statistical significance between $\mathrm{C}_{\max }, \mathrm{C}_{\min }$, and $\mathrm{AUC}$ as the driver for $\mathrm{E}-\mathrm{R}$ is likely due to the high correlation between the three exposure metrics in the Phase 2 trial. Though region was the only covariate in the

Fig. 4 Visual predictive check of DAS28 (CRP) exposure-response model - by fenebrutinib AUC bins at week 12. AUC, area under the concentration time curve. Black dots: observed responder fraction at fenebrutinib exposure quartiles; bands: $90 \%$ prediction interval; red middle lines: median prediction. longitudinal E-R model, region and baseline RF were correlated in the patient population evaluated (i.e. Latin America had higher baseline RF values). Therefore, it might be difficult to disentangle whether the covariate effect was truly due to region or other regional differences, such as baseline RF levels, which are important in the diagnosis and determination of RA severity (35). Interestingly, treatment history (MTX-IR vs. TNF-IR) was tested but was not a significant covariate in the E-R model, indicating similar E-R between MTX-IR and TNF-IR patients. This could possibly be due to the difference in background treatment or disease management between the two populations in this study. Ultimately, E-R in cohort 2 patients, who were TNF-IR, is unclear due to the small sample size and limited PK exposure range investigated.

\section{Model-Based Meta-Analysis (MBMA)}

Modeling of historical meta-analysis data sought to leverage publicly available information to enable direct and indirect

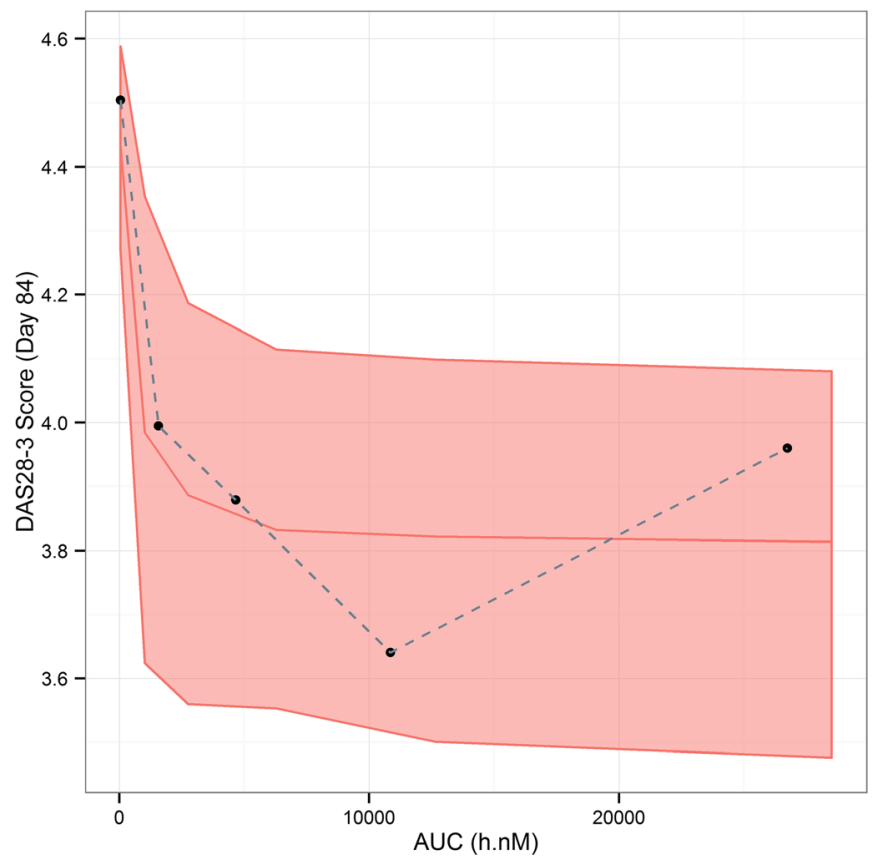



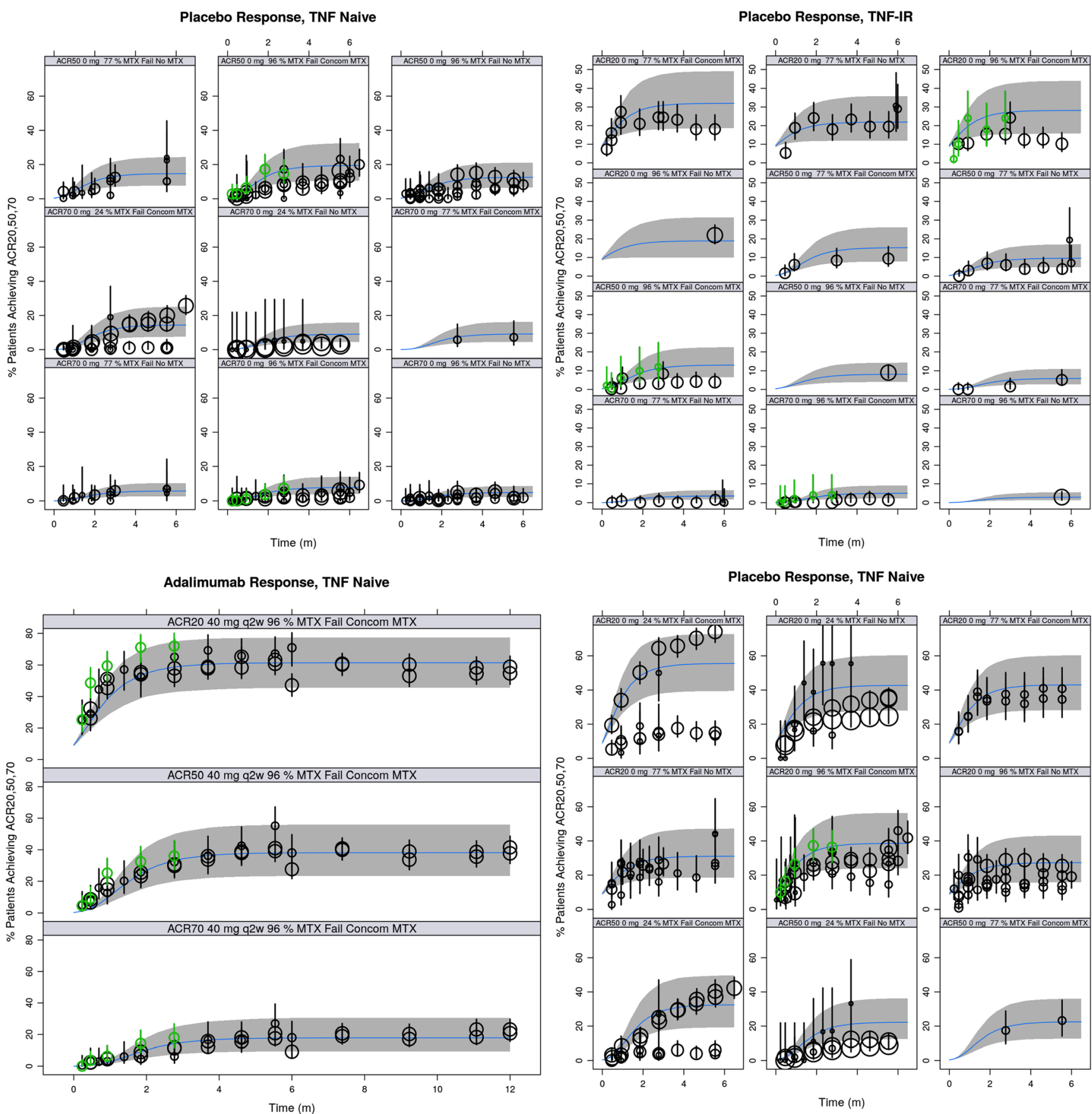

Fig. 5 Posterior predictive check for model-based meta-analysis model - time course by endpoint, dose, and patient subgroup for placebo and adalimumab treatments. TNF, tumor necrosis factor; IR, inadequate response, MTX, methotrexate; q2w, once every two weeks; concom, concomitant. black dots: observed responder fraction with symbol size scaled to sample size; black lines: $90 \%$ confidence interval for observations; green dots and lines: observed summary-level data and $90 \%$ confidence interval from fenebrutinib phase 2 trial; bands: $90 \%$ prediction interval; blue middle line: median prediction.

comparisons against competitors and between study populations. Such an analysis is particularly valuable at the end of Phase 2 milestone for informing go/no-go decisions to proceed to Phase 3 (36). First, MBMA aimed to quantitatively compare fenebrutinib against currently marketed therapies, through an indirect comparison of the performance of placebo and adalimumab historically versus that observed in the fenebrutinib Phase 2 trial. Second, MBMA sought to better inform the magnitude of impact of patient population covariates, with the initial interest focusing on informing the impact of having patients who were MTX-IR versus other patient populations, if possible.

Efficacy benchmarking against approved agents was focused on adalimumab and tofacitinib within this body of work, however, relative performance of other agents could also be compared using the established MBMA model. Tofacitinib is a janus kinase (JAK) inhibitor 
Fig. 6 Posterior predictive check for model-based meta-analysis model - time course of ACR50 for other drugs leveraged (selected doses). Note: only one dose level per treatment is shown as representative example. Black dots: observed responder fraction with symbol size scaled to sample size; black lines: $90 \%$ confidence interval for observations; bands: $90 \%$ prediction interval; blue middle line: median prediction.

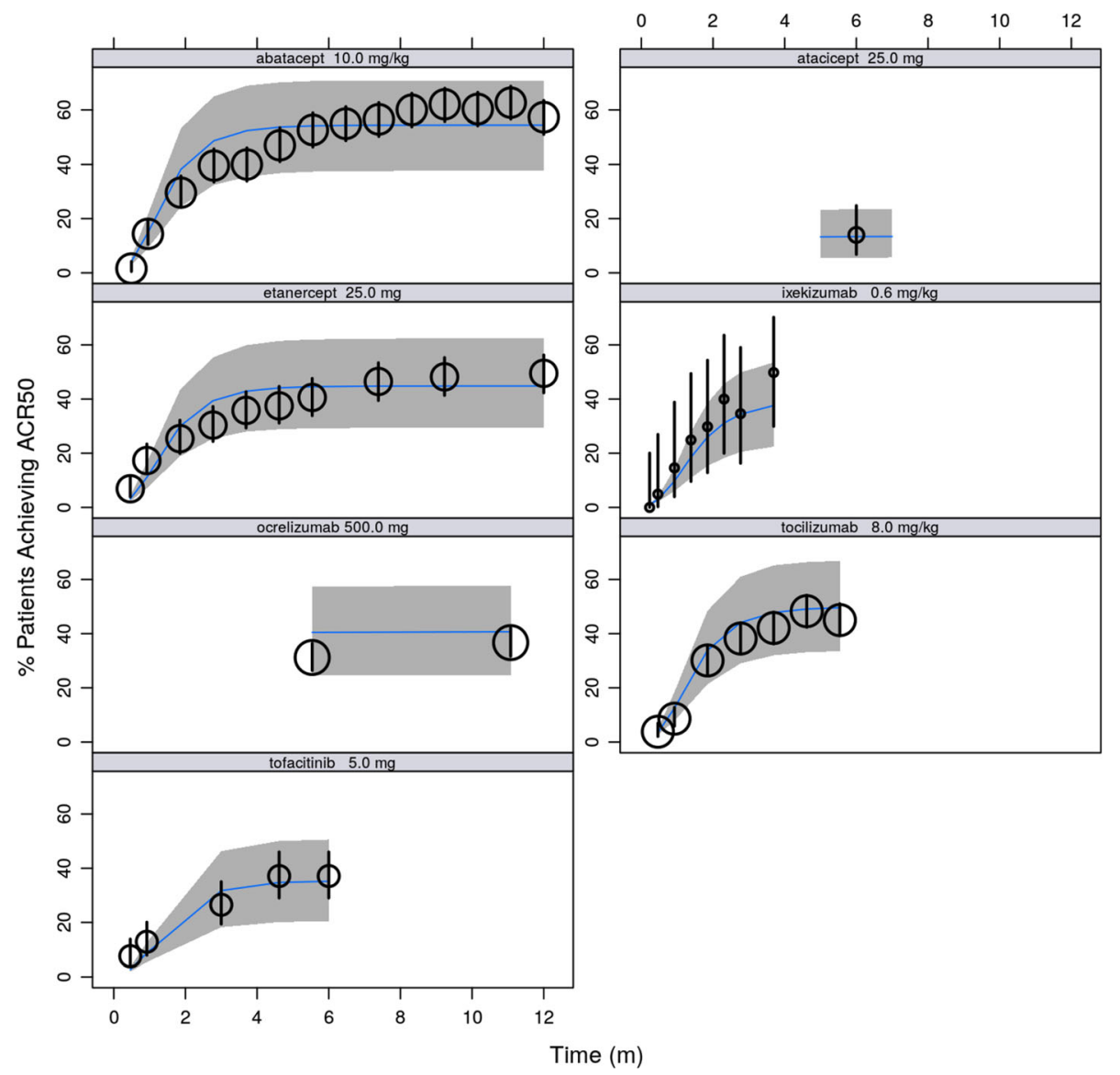

previously approved for the treatment in patients with an inadequate response to MTX, which was the target population in cohort 1 of the fenebrutinib Phase 2 trial as well. Further, covariates in the model could be used for clinical trial simulations in populations not yet investigated, permitting future decision makers to consider the merits of new or altered therapies far before committing large investments.

There was also a useful transfer of information from the E$\mathrm{R}$ analysis to the MBMA model development. Initially, it was difficult to obtain a stable estimate of the $\mathrm{ED}_{50}$ of fenebrutinib in the MBMA model. However, by leveraging the estimated $\mathrm{EC}_{50}$ from the E-R analysis, which is based on data from individual subjects, the final MBMA model that is based on summary-level data is much more informed in regard to the treatment effect of fenebrutinib.

The analysis had some limitations. Notably, efficacy of fenebrutinib beyond 12 weeks is unknown, limiting comparison at such observation times. Although MBMA analysis by Wang et al showed that ACR50 responses at 3 months after initiation of treatment is predictive of long term efficacy for most drug classes (37), this assumption might not apply to a molecule with a new mechanism of action and possibly a slower onset of action, and therefore longer monitoring to accurately determine maximum efficacy could be warranted.
Further, the MBMA had slightly different prediction results than observed (non-modeling) data from the fenebrutinib Phase 2 trial (24). It is hypothesized that the study patients from all arms are slightly over performing compared to median historical results for placebo and adalimumab treatments, although still within the variability of historical data. In our experience, MBMA can often yield slightly different results for an individual trial when it is modeled along with historical data, given the weight and richness of information from the latter.

\section{CONCLUSIONS}

The multiple analysis types as described herein provided an integrated view of model-based evidence, which has not been reported previously for a BTK inhibitor in patients with RA. This holistic approach yielded more insight than separate analyses alone, especially at an earlier phase of the drug development, where the uncertainly of treatment effect estimation and prediction could be large. Thus, the approach examines the totality of available evidence and allows better informed decision-making, and therefore is consistent with model-based strategies that are currently being evaluated by PDUFA VI to support drug development. 
Fig. 7 ACR20, ACR50, and ACR70 simulation for fenebrutinib (GDC-0853), adalimumab, and tofacitinib treatments in TNFinhibitor-naïve Population. TNF, tumor necrosis factor; MTX, methotrexate; bid, twice daily; q2w, once every two weeks. Bands: 90\% prediction interval; blue middle line: median prediction.
Drug Response, TNF Naive, 100\% MTX fail, Concomitant MTX

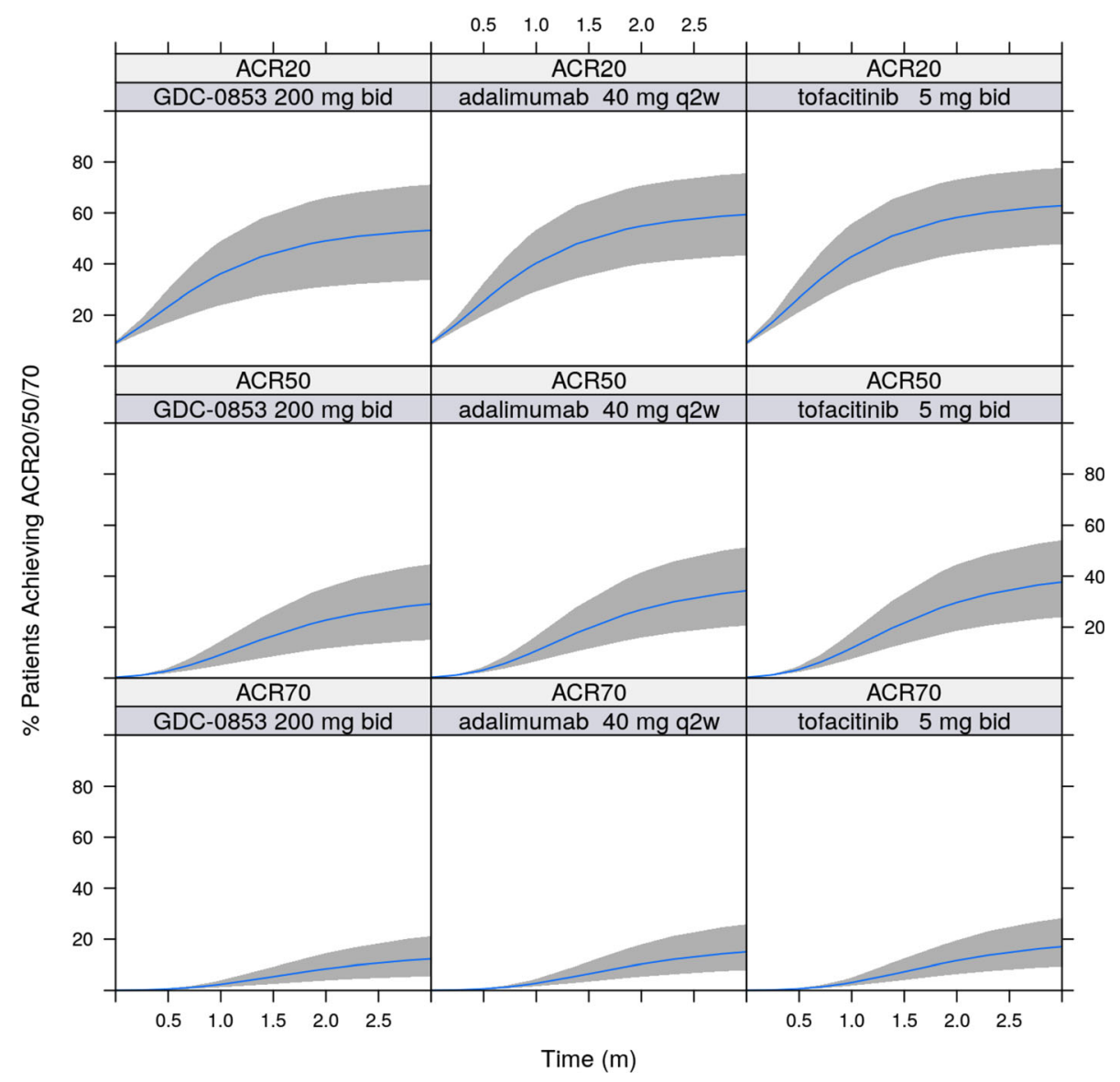

\section{ACKNOWLEDGEMENTS AND DISCLOSURES}

The authors would like to thank Hao Ding and Jiayi Han for their assistance with data preparation, Tamiko Katsumoto, Rui Zhao, and Jodie Peake for discussions on the efficacy analysis strategies, as well as Lee Hodge, Klaas Prins, Jason Chittenden, Oskar Alskär, and Fredrik Jonsson of qPharmetra for providing technical and writing support for the analyses, Certara USA Inc. and Excelra Knowledge Solutions Pvt. Ltd. for providing the meta-analysis databases, and Anshin BioSolutions, Inc., for editorial support of the manuscript.

Phyllis Chan, Jiajie Yu, Leslie Chinn, William Hanley, Katie Tuckwell, and Angelica Quartino are current or former employees of Genentech, Inc. (a member of the Roche group) and own or owned stock in F. Hoffman-La Roche. Marita Prohn, Jan Huisman, Brett Matzuka are salaried employees of qPharmetra, which was contracted by Genentech.

Open Access This article is licensed under a Creative Commons Attribution 4.0 International License, which permits use, sharing, adaptation, distribution and reproduction in any medium or format, as long as you give appropriate credit to the original author(s) and the source, provide a link to the
Creative Commons licence, and indicate if changes were made. The images or other third party material in this article are included in the article's Creative Commons licence, unless indicated otherwise in a credit line to the material. If material is not included in the article's Creative Commons licence and your intended use is not permitted by statutory regulation or exceeds the permitted use, you will need to obtain permission directly from the copyright holder. To view a copy of this licence, visit http://creativecommons.org/licenses/by/4.0/.

\section{REFERENCES}

1. FDA. PDUFA Reauthorization performance goals and procedures Fiscal years 2018 through 2022 [Available from: https://www.fda. gov/media/99140/download].

2. Mould DR. Model-based meta-analysis: an important tool for making quantitative decisions during drug development. Clin Pharmacol Ther. 2012;92(3):283-6.

3. Scott DL, Wolfe F, Huizinga TW. Rheumatoid arthritis. Lancet. 2010;376(9746):1094-108.

4. Wang W, Zhou H, Liu L. Side effects of methotrexate therapy for rheumatoid arthritis: a systematic review. Eur J Med Chem. 2018;158:502-16. 
5. Kalden JR, Schulze-Koops H. Immunogenicity and loss of response to TNF inhibitors: implications for rheumatoid arthritis treatment. Nat Rev Rheumatol. 2017;13(12):707-18.

6. Genovese MC, Fleischmann R, Combe B, Hall S, Rubbert-Roth A, Zhang Y, et al. Safety and efficacy of upadacitinib in patients with active rheumatoid arthritis refractory to biologic disease-modifying anti-rheumatic drugs (SELECT-BEYOND): a double-blind, randomised controlled phase 3 trial. Lancet. 2018;391(10139):2513-24.

7. Burmester GR, Kremer JM, Van den Bosch F, Kivitz A, Bessette L, Li Y, et al. Safety and efficacy of upadacitinib in patients with rheumatoid arthritis and inadequate response to conventional synthetic disease-modifying anti-rheumatic drugs (SELECT-NEXT): a randomised, double-blind, placebo-controlled phase 3 trial. Lancet. 2018;391(10139):2503-12.

8. van Vollenhoven RF, Fleischmann R, Cohen S, Lee EB, Garcia Meijide JA, Wagner S, et al. Tofacitinib or adalimumab versus placebo in rheumatoid arthritis. N Engl J Med. 2012;367(6):50819.

9. Taylor PC, Keystone EC, van der Heijde D, Weinblatt ME, Del Carmen ML, Reyes Gonzaga J, et al. Baricitinib versus placebo or Adalimumab in rheumatoid arthritis. N Engl J Med. 2017;376(7): 652-62.

10. Emery P, Keystone E, Tony HP, Cantagrel A, van Vollenhoven R, Sanchez A, et al. IL-6 receptor inhibition with tocilizumab improves treatment outcomes in patients with rheumatoid arthritis refractory to anti-tumour necrosis factor biologicals: results from a 24-week multicentre randomised placebo-controlled trial. Ann Rheum Dis. 2008;67(11):1516-23.

11. Gabay C, Msihid J, Zilberstein M, Paccard C, Lin Y, Graham $\mathrm{NMH}$, et al. Identification of sarilumab pharmacodynamic and predictive markers in patients with inadequate response to TNF inhibition: a biomarker substudy of the phase 3 TARGET study. RMD Open. 2018;4(1):e000607.

12. Vanhoutte F, Mazur M, Voloshyn O, Stanislavchuk M, Van der Aa A, Namour F, et al. Efficacy, safety, pharmacokinetics, and pharmacodynamics of Filgotinib, a selective JAK-1 inhibitor, after shortterm treatment of rheumatoid arthritis: results of two randomized phase IIa trials. Arthritis Rheumatol. 2017;69(10):1949-59.

13. Satterthwaite AB, Li Z, Witte ON. Btk function in B cell development and response. Semin Immunol. 1998;10(4):309-16.

14. Schmidt U, Boucheron N, Unger B, Ellmeier W. The role of Tec family kinases in myeloid cells. Int Arch Allergy Immunol. 2004;134(1):65-78.

15. Brunner C, Muller B, Wirth T. Bruton's tyrosine kinase is involved in innate and adaptive immunity. Histol Histopathol. 2005;20(3): 945-55.

16. Di Paolo JA, Huang T, Balazs M, Barbosa J, Barck KH, Bravo BJ, et al. Specific Btk inhibition suppresses B cell- and myeloid cellmediated arthritis. Nat Chem Biol. $2011 ; 7(1): 41-50$.

17. Satterthwaite AB, Witte ON. The role of Bruton's tyrosine kinase in B-cell development and function: a genetic perspective. Immunol Rev. 2000;175:120-7.

18. So L, Fruman DA. PI3K signalling in B- and T-lymphocytes: new developments and therapeutic advances. Biochem J. 2012;442(3): 465-81.

19. Puri KD, Di Paolo JA, Gold MR. B-cell receptor signaling inhibitors for treatment of autoimmune inflammatory diseases and B-cell malignancies. Int Rev Immunol. 2013;32(4):397-427.

20. Katewa A, Wang Y, Hackney JA, Huang T, Suto E, Ramamoorthi $\mathrm{N}$, et al. Btk-specific inhibition blocks pathogenic plasma cell signatures and myeloid cell-associated damage in IFNalpha-driven lupus nephritis. JCI Insight. 2017;2(7):e90111.

21. Crawford JJ, Johnson AR, Misner DL, Belmont LD, Castanedo G, Choy R, et al. Discovery of GDG-0853: a potent, selective, and noncovalent Bruton's tyrosine kinase inhibitor in early clinical development. J Med Chem. 2018;61(6):2227-45.
22. Herman AE, Chinn LW, Kotwal SG, Murray ER, Zhao R, Florero $\mathrm{M}$, et al. Safety, pharmacokinetics, and pharmacodynamics in healthy volunteers treated with GDC-0853, a selective reversible Bruton's tyrosine kinase inhibitor. Clin Pharmacol Ther. 2018;103(6):1020-8.

23. Byrd JC, Smith S, Wagner-Johnston N, Sharman J, Chen AI, Advani R, et al. First-in-human phase 1 study of the BTK inhibitor GDC-0853 in relapsed or refractory B-cell NHL and CLL. Oncotarget. 2018;9(16):13023-35.

24. Cohen S, Tuckwell K, Katsumoto TR, Zhao R, Lee C, Berman A, et al. OP0025 Fenebrutinib compared to placebo and adalimumab in patients with inadequate response to either methotrexate therapy or prior TNF therapy: phase 2 study. Ann Rheum Dis. 2019;78(Suppl 2):80-1.

25. Morimoto A, Rae J, Chinn L, Ramamoorthi N, Hwang O, Ward A, et al. FRI0129 the BTK inhibitor, fenebrutinib, effectively modulates B and myeloid cell biology in rheumatoid arthritis patients. Ann Rheum Dis. 2019;78(Suppl 2):733-4.

26. Bergstrand M, Hooker AC, Wallin JE, Karlsson MO. Predictioncorrected visual predictive checks for diagnosing nonlinear mixedeffects models. AAPS J. 2011;13(2):143-51.

27. Anderson J, Caplan L, YazdanyJ, Robbins ML, Neogi T, Michaud $\mathrm{K}$, et al. Rheumatoid arthritis disease activity measures: American College of Rheumatology recommendations for use in clinical practice. Arthritis Care Res (Hoboken). 2012;64(5):640-7.

28. FDA. Guidance for Industry: Rheumatoid Arthritis: Developing Drug Products for Treatment [Available from: https://www.fda. gov/media/86066/download].

29. Lacroix BD, Karlsson MO, Friberg LE. Simultaneous exposureresponse modeling of ACR20, ACR50, and ACR70 improvement scores in rheumatoid arthritis patients treated with Certolizumab Pegol. CPT Pharmacometrics Syst Pharmacol. 2014;3:e143.

30. Yano Y, Beal SL, Sheiner LB. Evaluating pharmacokinetic/ pharmacodynamic models using the posterior predictive check. J Pharmacokinet Pharmacodyn. 2001;28(2):171-92.

31. Demin I, Hamren B, Luttringer O, Pillai G, Jung T. Longitudinal model-based meta-analysis in rheumatoid arthritis: an application toward model-based drug development. Clin Pharmacol Ther. 2012;92(3):352-9.

32. Savic RM, Jonker DM, Kerbusch T, Karlsson MO. Implementation of a transit compartment model for describing drug absorption in pharmacokinetic studies. J Pharmacokinet Pharmacodyn. 2007;34(5):711-26.

33. Maringwa J, Kagedal M, Hamren UW, Martin P, Cox E, Hamren B. Pharmacokinetic-pharmacodynamic modeling of fostamatinib efficacy on ACR20 to support dose selection in patients with rheumatoid arthritis (RA). J Clin Pharmacol. 2015;55(3):328-35.

34. Lamba M, Hutmacher MM, Furst DE, Dikranian A, Dowty ME, Conrado D, et al. Model-informed development and registration of a once-daily regimen of extended-release Tofacitinib. Clin Pharmacol Ther. 2017;101(6):745-53.

35. Pike RM, Sulkin SE, Coggeshall HC. Serological reactions in rheumatoid arthritis; factors affecting the agglutination of sensitized sheep erythrocytes in rheumatid-arthritis serum. J Immunol. 1949;63(4):441-6.

36. Upreti VV, Venkatakrishnan K. Model-based meta-analysis: optimizing research, development, and utilization of therapeutics using the totality of evidence. Clin Pharmacol Ther. 2019;106(5):981-92. https://doi.org/10.1002/cpt.1462.

37. Wang Y, Zhu R, Xiao J, Davis JC Jr, Mandema JW, Jin JY, et al. Short-term efficacy reliably predicts long-term clinical benefit in rheumatoid arthritis clinical trials as demonstrated by modelbased meta-analysis. J Clin Pharmacol. 2016;56(7):835-44.

Publisher's Note Springer Nature remains neutral with regard to jurisdictional claims in published maps and institutional affliations. 